\title{
New markers for the detection of polycystic ovary syndrome
}

\begin{abstract}
Polycystic Ovary Syndrome (PCOS) is a highly prevalent, complex genetic disorder of the endocrine system in women. Alterations that occur in women with PCOS can be due to several predisposing factors; among these contributors are genetic and epigenetic variations. Environmental factors play a weaker role, mainly in worsening insulin resistance. Enzyme, protein and genetic markers can depend as a biochemical diagnosis of PCOs. The genetic markers have been identified to be related to PCOS wasn't useful for early diagnosis, which can only be used to confirm PCOS in patients already exhibiting the definitive symptoms. Protein and enzyme markers are commonly used for prognosis and monitoring the patient to prevent the development of the complications of PCOS. Proteins of the adipose tissue have been found to be greatly related to insulin resistance and the development of PCOS. The nature of enzymes and proteins of instability and easily degradable have prevented sufficient research from being carried out on them. Therefore, the diagnosis of PCOS relies on the analysis of multiple factors.
\end{abstract}

Keywords: ovary syndrome, biochemical diagnosis, fertility, ovulation, reproduction
Volume 10 Issue 4 - 2019

\author{
Elham Sharif, Mariam Alwakeel \\ Department of Biomedical Sciences, College of Health Sciences, \\ Qatar University, Qatar
}

Correspondence: Dr. Elham Sharif, Department of Biomedical Sciences, College of Health Science, Qatar University, Po box 2713, Doha Qatar,Tel +97431002102, Email e.sharif@qu.edu.qa

Received: June 25, 2019| Published: July 15, 2019
Abbreviations: PCOS, polycystic ovary syndrome; IRS1, insulin receptor substrate 1; SHBG, sex hormone-binding globulin; TNF, tumor necrosis factor; IL-6, interleukin-6; LH, luteinizing hormone; FSH, follicle stimulating hormone; GnRH, gonadotropinreleasing hormone; $\mathrm{AMH}$, anti-müllerian; $\mathrm{BPA}$, bsphenol A

\section{Introduction}

Polycystic Ovary Syndrome (PCOS) is a highly prevalent, complex genetic disorder of the endocrine system in women. There have been various criteria suggested for diagnosis, although there are 2 most accepted criteria: as suggested by the $1990 \mathrm{NIH}$ - National Institute of Child Health and Human Development Conference of PCOS, where the presence of hyperandrogenism, hyperandrogenemia and oligoovulation, followed by exclusion of any other disorder is considered diagnostic for PCOS, or as suggested by the broader 2003 Rotterdam consensus, in which presence of any 2 of the 3 abovementioned symptoms, followed by exclusion of other endocrinopathies is considered diagnostic for PCOS. Following the NIH criteria, about $10 \%$ of women globally are diagnosed with PCOS, while the Rotterdam criteria gives a higher percentage. ${ }^{1,2}$ This makes PCOS the most common human disorder and the most common single disorder of reproductive women. Although it was previously assumed that PCOS had relevance only in cases of fertility, it has now been shown to cause various long-term risks, such as hyperinsulinism, diabetes mellitus, cardiovascular disease and other morbidities. ${ }^{3,4}$

\section{Pathophysiology}

Alterations that occur in women with PCOS can be due to several predisposing factors; among these contributors are genetic and epigenetic variations. Environmental factors play a weaker role, mainly in worsening insulin resistance.

This complex metabolic disorder has no definitive pathway for explaining all the internal and external symptoms associated. However, studies have revealed a general understanding of the pathophysiology of the disorder. In PCOS patients, the primary defects are seen from two perspectives in the body, insulin resistance and relative LH/FSH levels. ${ }^{5}$ Rhesus monkeys and sheep were used as animal models of PCOS by exposing them to elevated levels of androgens during gestation. Evidence from these models suggested that developmental programming by androgen has a role in the deposition of abdominal fat and developing resistance to insulin. ${ }^{6}$ Many PCOS patients may develop insulin resistance; which means that the cells in their bodies fail to respond to the insulin hormone, and therefore, fails to take up the glucose. Since glucose is not utilized for energy production, it is stored in the body, resulting in weight gain. Eventually, excess insulin in the body will stimulate the ovaries to increase androgen production. This leads to oligo-ovulation (irregular ovulation) or anovulation (the absence of ovulation). Some typical insulin target organs, such as adipose tissue and muscles, are affected by insulin resistance, which makes it tissue-selective. Serine phosphorylation of the insulin receptor and of insulin receptor substrate 1 (IRS1) is markedly increased in muscles, causing a dysfunction in insulin signaling and in activation of mitogen-activated protein kinases MEK1 and MEK2. ${ }^{7}$ Other organs like the ovary are spared and not affected by insulin resistance.

Insulin resistance in PCOS is also associated with an increase of testosterone bioactivity by reducing the synthesis of sex hormonebinding globulin (SHBG) in the liver (which is inhibited by insulin). ${ }^{5}$ It is very likely among women with PCOS to have slight differences in fat distribution. According to studies, strong evidence indicates that adipocytes are abnormal in those patients, promoting insulin resistance and subclinical inflammation. For instance, adipocytes derived from patients with PCOS tend to have suppressed insulinmediated glucose transport by the action of inflammatory cytokines such as tumor necrosis factor (TNF) and Interleukin-6 (IL-6). Women with PCOS appear to have enlarged adipocytes, lower lipoprotein lipases activity and impaired catecholamine-induced lipolysis.

Furthermore, the two main pituitary hormones that orchestrate major changes in the body are Luteinizing hormone (LH), and Follicle stimulating hormone (FSH). These hormones are essential 
for reproduction. LH stimulates the production of androgen substrates which are then converted to create sex hormones such as testosterone and estrogen. In normal situations, synchronized signals between gonadotropins and factors produced in the developing follicle of the ovary result in ovulation. In women with PCOS, the growth of the follicle is arrested at an early antral stage, this results in follicular maturation arrest, accumulation of small antral follicles within the periphery of the ovary, giving it the polycystic morphology. FSH stimulates the growth of the ovarian follicle to allow maturation of the egg before it releases. It is important for FSH and LH to be in precise balance to avoid complications. Normally, during the beginning of menstruation cycle, FSH is higher than LH. However, in PCOS, this is reversed, and the release of gonadotropin-releasing hormone $(\mathrm{GnRH})$ from the hypothalamus is often disturbed leading to hypersecretion of $\mathrm{LH}^{4}$ Initially, instead of a higher FSH, there is an increase of the level of $\mathrm{LH}$ which produces an excess of androgen substrates that leads to elevated testosterone levels, which is associated with excess body and facial hair.

Although serum follicle-stimulating hormone levels remain normal, follicles appear to be more resistant to FSH in PCOS patients. This could be due to an increase of intra-ovarian and anti-müllerian (AMH) hormone levels; high levels of AMH inhibits FSH action as well as recruitment of more primordial follicles. ${ }^{4}$ Thus, the ovaries are not stimulated adequately for maturation, and eventually ovulation. This is the reason why women with PCOS have difficulty conceiving and experience irregular menstruation cycles.

Moreover, the observed increased production of androgen in PCOS is primarily due to stimulated synthesis of the hormone by follicular theca cells, which show high expression of multiple genes encoding steroidogenic enzymes. ${ }^{8}$ Further evidence supports that in some patients with PCOS, excess ovarian androgen is genetically determined. This has been reported after conducting some studies on theca cells obtained from patients and observing that DENND1A, a candidate gene, was overexpressed.

It is also observed in PCOS patients that the gene that encodes for the rate-limiting enzyme (CYP17A1) is elevated in theca cells obtained from patients. Those cells are more responsive to insulin and LH in terms of androgen secretion in patients rather than cells obtained from healthy individuals. In addition, reducing hepatic synthesis of SHBG due to hyperinsulinemia contributes to hyperandrogenism, which in turn leads to increased testosterone fractions. Adrenocortical dysfunction is also suggestive of hyperandrogenism as a secondary source along with excess androgen from ovaries as the primary source.

Hirsutism is a consequence of excess androgen secretion. Because of testosterone and dihydrotestosterone stimulation of ornithine decarboxylase synthesis in the hair follicle, this stimulates the production of polyamine. These amines are necessary for cellular proliferation, including hair growth in the follicles. ${ }^{7}$

As for the environmental factors, expression of PCOS is affected by lifestyle changes. For instance, greater body weight and dietary saturated fat intake worsens metabolic and reproductive abnormalities. However, losing weight lowers androgen and insulin circulating levels, while enhancing hirsutism, as well as ovulatory dysfunction. ${ }^{9}$

Another factor could be endocrine disrupting chemicals, which can alter the metabolic and ovarian functions, resulting in PCOS-like symptoms. One of these chemical that is detected in PCOS patients is Bisphenol A (BPA), an estrogenic industrial plasticizer. Accumulation of BPA in susceptible women might increase the severity of PCOS phenotype expression. ${ }^{10}$

\section{PCOS markers}

The definition and diagnosis of PCOS varies from lab to lab, and country to country. While the European criteria for diagnosis depend upon an abnormal appearance of the ovaries, the North American criteria for diagnosis depend upon various biochemical evidences of hyperandrogenism/hyperandrogenemia.

The biochemical diagnosis can depend upon enzyme, protein and genetic markers. In this section, the various markers used for PCOS diagnosis, their advantages and disadvantages are discussed.

Studies have shown a strong correlation between genetic factors and developing PCOS, and it shows an autosomal dominant inheritance scheme. Usually, there is a $30-50 \%$ chance that a woman might develop PCOS if one of her family members is affected. Twin studies indicate that monozygotic twin sisters have twice as high chance of developing PCOS as dizygotic twins. Up until now, researchers have identified around 100 susceptibility genes associated with PCOS. ${ }^{4}$ However, many studies indicate that it may have ontogenetic characteristics. Even though inheritance shows a strong familial relationship, the phenotypic manifestation among same family members may differ. ${ }^{11}$ According to Shaikh, Dadachanji, and Mukherjee (2014), the genetic factor in PCOS is not the only agent that contributes to this disease; environmental factors and other abnormalities also play a role in the development of this disease. PCOS also shows a strong relationship with many other disorders, like hyperinsulinemia and hyperandrogenemia, which tells us that it is a heterogenic syndrome. Because of this, the investigation on PCOS genetic background needs the knowledge of other disorder's molecular basis. ${ }^{12}$ PCOS has been assumed to be associated with various genes regulating insulin function and androgen synthesis.

One of the most important correlated diseases is insulin resistance, which is described as the inability of host cells to respond to insulin secretion. A brief look at insulin function suggests that it is involved in a complex cascade that activates various cellular receptors, including INSR and IGF-1 receptors. INSR is a major insulin receptor than has a tetrameric structure consisting of two alpha and two beta subunits. The activation of INSR is mediated mainly by insulin which triggers tyrosine kinase activation, which in turn activates a long complex signaling cascade. A study that was done by Urbanek, et al., ${ }^{13}$ on 37 PCOS candidates' genes showed that there is post signaling defect of INSR in their skeletal muscles and adipose tissues. One of the markers that showed a strong association with INSR defect and associated with PCOS is D19S884, which lies on chromosome 19 P13.2. ${ }^{13}$

Another molecule that is correlated with INSR activation is IRS. The phosphorylation of IRS allows INSR to communicate with insulin signaling mediators like PI3K, Fyn and Crk. A study that was done by Dunaif, Wu, Lee, and Diamanti-Kandarakis, ${ }^{10}$ showed that PCOS candidates have defect in their IRS molecules in their skeletal muscles. ${ }^{14}$ Studies also showed that the polymorphisms in IRS-1 and IRS-2 are associated with many PCOS cases, especially in women from Italy, Japan, Greek, Turkey and other different populations.

ENPP1, Calpain-10 and PPAR $\gamma$ are also other molecules associated with PCOS that mediate insulin signaling. The complication with these markers is polymorphism. According to the study above, these 
markers did not manifest in all PCOS cases, and their exact role in PCOS pathogenesis is not yet understood properly.

As indicated earlier, PCOS is associated with many other disorders, as there are many studies which describe the influence of other disorders in PCOS' pathogenesis.

Even though PCOS shows a variety of symptoms, the main characteristic is defects in hormonal secretion, i.e., there is an abnormal quantity of male hormones which leads to faults in menstruation and the formation of cyst on ovaries. To understand the genetic basis of PCOS, it is important to investigate the abnormalities in steroidogenesis in the molecular level.

One of the diagnostic features of PCOS, which is the abnormal elevation of circulating androgen, is due to the overexpression of theca cells in ovaries which are responsible for the production of androstenedione and testosterone. This is due to the increased transcription and mRNA stability of steroidogenic enzymes.

Historically, CYP17 has showed a correlation with hyperandrogenism. It transcribes $\mathrm{P} 450 \mathrm{c} 17 \alpha$ enzyme, which is the limiting step in androgen synthesis. According to a study of Rosenfield et al (1999), patients with hyperandrogenism and PCOS have an elevated P450c17 $\alpha$ activity which may correlate with the massive androgen secretion. CYP17 is located on chromosome 10q24.3. Some studies suggest that the polymorphism of CYP17 may influence PCOS manifestation, but other studies failed to confirm this association. To date, the exact role of this gene has not been defined.

Another gene, CYP11A is considered as a candidate gene for PCOS and hyperandrogenism. CYP11A gene involved in the initial step of androgenesis; it encodes for the enzyme that cleaves the side chain of cholesterol, and prepares it for conversion into androgen. A polymorphism in this gene is defined by repeats of (tttta) at 528bp at CYP11A promoter. This genotype has been strongly associated with PCOS in 20 pedigree that express the disease. ${ }^{15}$

Although these genes showed a relationship with PCOS, many of them failed to be reliable in early diagnosis of PCOS. This is due to the absence of standard expression scheme among the population, and the presence of other genes that influence the expression of hyperandrogenism.

A recent study by Eisenberg et al. ${ }^{16}$ showed the possibility to screen the anovulatory women using serum miRNA20b and miRNA-429. The study involved 7 normally ovulating women, 15 normally ovulating women with pure male infertility factor, and 18 women with PCOS. The method of detection was RT-PCR. The results showed that women with PCOS and anovulatory conditions showed higher concentration of miRNA20b and miRNA-429 than spontaneously ovulating women. ${ }^{16}$ miRNA play a role in the formation of the primordial follicles and regulate follicular development. It shows different concentration during the luteal cycles. miRNA can regulate gene expression by binding to the 3 ' in the untranslated region of mRNA. This inhibits translation by making the target mRNA unstable. The exact role of miRNA in PCOS is not defined yet. However, a significant increase in specific miRNA has been manifested in follicular fluids and patients' serum. A study tried to explain miRNA role in PCOS using rat model expression. The study used DHT to mimic hyperandrogenic levels in human. The analysis of the ovaries in these models showed hyper elevation of 72 miRNA compared to the control, where 17 miRNA showed depression. Another study explained miRNA role in regulation apoptosis and differentiation in ovaries cells, and that may correlate with abnormal miRNA incidence in PCOS. ${ }^{17}$

Zao et al (2017) indicated that there could a relationship between the methylation of o-PPARGC1A promoter in leukocytes and mitochondrial DNA of PCOS patient. The cross - sectional study indicates that there is an elevation in the concentration of methylated PPARGC1A promoter in PCOS patient compared to normal control. It also indicates that there is depletion in mitochondrial DNA in PCOS compared to healthy women. The study suggests that there could be a relation between these markers and the metabolic abnormalities of PCOS patients. This suggestion is enhanced by the fact that most PCOS patients who had high methylated o-PPARGC1A promoter had high BMI too. Moreover, the study suggests that these markers associate with insulin and IRN concentration not only in PCOS, but also in nonPCOS women. One of the explanations suggested by the study about the elevation of methylated PPARGC1A promoter in leukocytes is epigenetics. Androgen enhances the epigenetic alternation of patient genome, which inevitably causes hypermethylation of PPARGC1A promoters. PPARGC1A promoter in leukocytes and mitochondrial DNA play a remarkable role in human physiological process, including the maintenance of blood pressure and cholesterol regulation. Thus, the ratio of PPARGC1A promoter in leukocytes and mitochondrial DNA can be used as marker for metabolic risk in PCOS.

An enclosure of the genes most correlated with PCOS is not impossible. A study done by Panda, P. K., Rane, R., Ravichandran, R., Singh, S., \& Panchal, H. (2016) indicated that there are 43 genes that are related to PCOS (Table 1). ${ }^{11}$

Recently some studies candidate probable markers for PCOS that may be used in future as definitive marker for screening and diagnosis. One of these markers is D19S884 marker which showed a high specificity to PCOS. It is a microsatellite that lies in 55 intron in fibrillin gene. The fibrillin gene functions on TGF-B regulation; however, their exact role in PCOS is not defined yet.

Another important gene is TCF7L2, which lies in type 2 diabetes locus. IRSN gene has also showed a strong relation with PCOS. For screening purposes, miRNA has shown to be of great value to detect anovulation patient; however, it can only define that there is a defect in patient ovulation cycle. ${ }^{11}$

As mentioned earlier, PCOS is a complex syndrome that correlates with many disorders, many of which arise from genetic backgrounds, especially insulin receptors abnormalities. However, due to many factors including polymorphism, environmental factors, and variation of causative agent in PCOS, the diagnostic value of these genetic markers may be useless in many cases. Many studies tried to find a link between PCOS and specific genes in patient's genomes, while others tried to utilize the linkage studies to demonstrate a precise diagnostic tool for the disease. Some of the studies appeared successful in finding a significant marker, while others improved that this marker has no specificity or any important role in testing. This can be related to the different sensitivity and specificity used for the test. For instance, the $P O M C$ gene, rs12473543, was previously thought to be related with PCOS but after correction testing, it showed no significant value. 
Table I a summary of 43 genes that are found in PCOS patients including their accession name, PDB-ID and Unirprot ID

\begin{tabular}{|c|c|c|c|c|c|}
\hline \multicolumn{6}{|c|}{ List of 43 Genes involved in PCOS with UNIPROT-ID. PDB-ID and chromosomal locations } \\
\hline Gene name & Locus & Organism & Uniprot ID & Accession number & PDB-ID \\
\hline \multirow[t]{2}{*}{$\begin{array}{l}\text { P450 side-chain } \\
\text { cleavage enzyme } \\
\text { (Marker Locus:0I5 } \\
\text { 3520) }\end{array}$} & $\begin{array}{l}\text { Chromosome 15: } \\
74,367,990-74,368,179\end{array}$ & Homo & P05I08 & P05I08 & $3 N 9 Y$ \\
\hline & & Sapiens & & & \\
\hline \multirow[t]{2}{*}{$\begin{array}{l}\text { Sex hormone binding } \\
\text { globulin }\end{array}$} & $\begin{array}{l}\text { Chromosome 17: } \\
7,613,946-7,633: 383\end{array}$ & Homo & PO4278 & ABY68008 & 1028 \\
\hline & & Scptens & & & \\
\hline \multirow[t]{2}{*}{$\begin{array}{l}\text { Luteiniaing hormone } \\
\text { choriogonadotropin } \\
\text { receptor }\end{array}$} & $\begin{array}{l}\text { Chromosome 2: } \\
48,708,77048.723,514\end{array}$ & Homo & P22888.4 & P22888 & ILUT \\
\hline & & Sapiens & & & \\
\hline \multirow[t]{2}{*}{$\begin{array}{l}\text { Follicle-stimulating } \\
\text { hormone receptor }\end{array}$} & $\begin{array}{l}\text { Chromosome 2: } \\
48,962,15749,154,537\end{array}$ & Homo & P23945 & AAB26480 & IXUN \\
\hline & & Sapiens & & & \\
\hline \multirow[t]{3}{*}{$\begin{array}{l}\text { Mothers against } \\
\text { decapentaplegic } \\
\text { homolog } 4\end{array}$} & $\begin{array}{l}\text { Chromosome 18: } \\
\text { 5I,028:394-51,085,045 }\end{array}$ & Homo & 13485 & NP 005350 & IDDI \\
\hline & & Sapiens & & & \\
\hline & Chromosome & & & & \\
\hline \multirow[t]{3}{*}{ Leptin } & $\begin{array}{l}7:|28,24|: 284- \\
\mid 28,257,628\end{array}$ & Homo & P4II59 & AAH69452 & I_AX8 \\
\hline & & \&Ver. & & & \\
\hline & Chromosome & & & & \\
\hline \multirow[t]{3}{*}{ Leptin receptor } & $\begin{array}{l}1: 65,420,652- \\
65,641,559\end{array}$ & Homo & P48357 & AAB09673 & $3 \mathrm{~V} 60$ \\
\hline & & \&piens & & & \\
\hline & Chromosome & & & & \\
\hline \multirow[t]{2}{*}{ Inhibin beta-B } & $\begin{array}{l}2: 120,346,143- \\
120,351,808\end{array}$ & Homo & P09529 & АAH30029 & \\
\hline & & Sapiens & & & \\
\hline \multirow[t]{2}{*}{ Inhibin beta-A } & $\begin{array}{l}\text { Chromosome 7: } \\
41,685,114-41,703,108\end{array}$ & Homo & P08476 & AAH07858 & INYS \\
\hline & & Sapiens & & & \\
\hline \multirow[t]{2}{*}{$\begin{array}{l}\text { Inhibits A (gene symbol: } \\
\text { INHA) }\end{array}$} & $\begin{array}{l}\text { Chromosome 2, 219,5 } \\
69: 162-219,575,713\end{array}$ & Homo & P05III & P05III & \\
\hline & & \&pi-ens & & & \\
\hline Pro-opiomelanecortin & $\begin{array}{l}\text { Chromosome / } \\
25,160,853-25,168,903\end{array}$ & Homo & P0II89 & P0II89 & \\
\hline (gene symbol: POMC). & & Segal. & & & \\
\hline $\begin{array}{l}\text { Uncoupling protein } \\
213\end{array}$ & $\begin{array}{l}\text { Chromosome II: } \\
73,974,667-73,983,307\end{array}$ & Homo & P5585I & P5585I & \\
\hline $\begin{array}{l}\text { (gene symbol: UCP2 } \\
+3 \text { ) }\end{array}$ & & Sapiens & & & \\
\hline
\end{tabular}


Table Continued

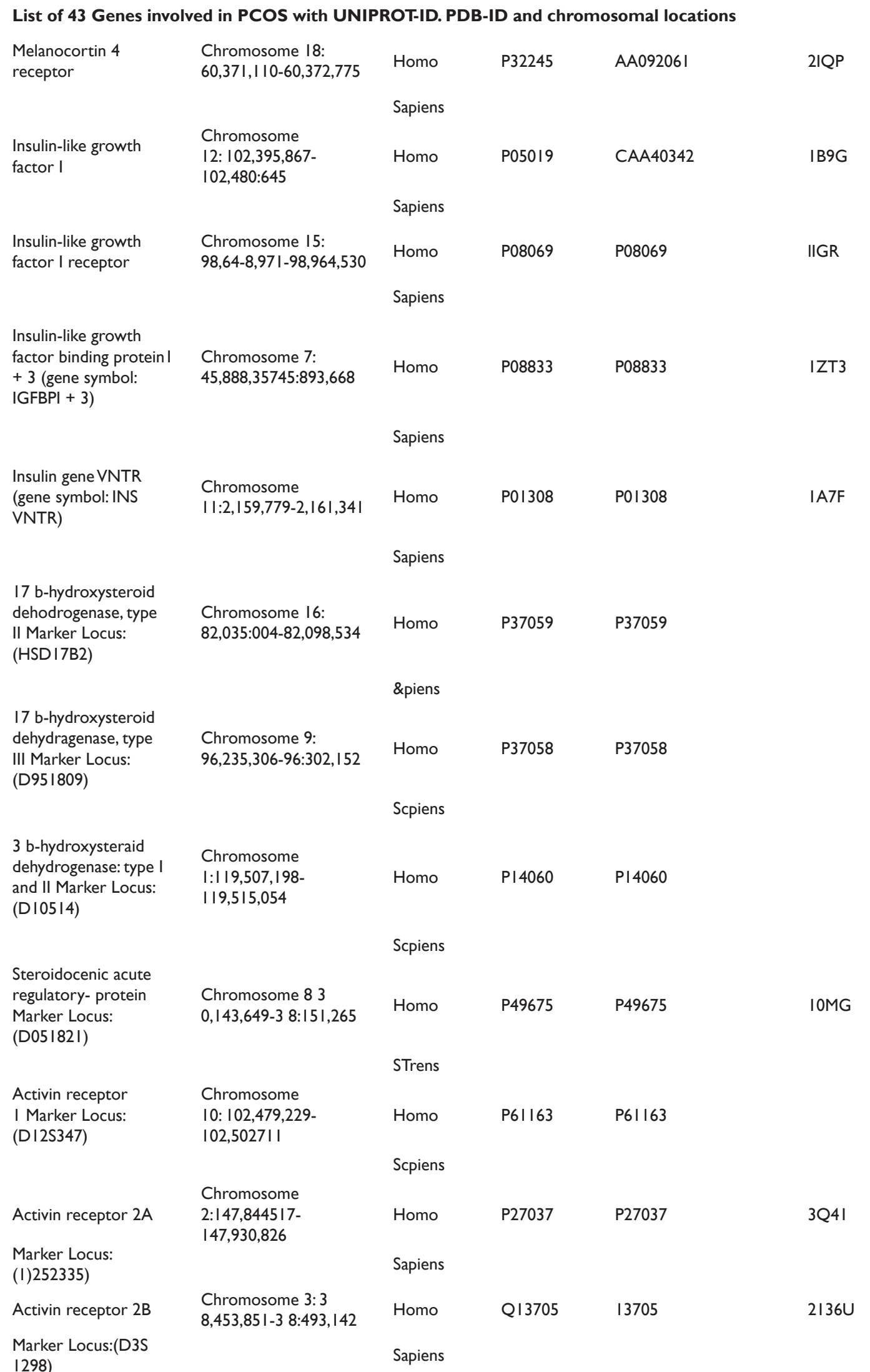




\begin{tabular}{|c|c|c|c|c|c|}
\hline $\begin{array}{l}\text { Inhibin C Marker } \\
\text { Locus: (DI20169I) }\end{array}$ & $\begin{array}{l}\text { Chromosome } 12 \text { NC } \\
00001112\end{array}$ & Homo & P55I03 & P55I03 & \\
\hline & $(5743468557452060)$ & Scpiens & & & \\
\hline \multirow[t]{2}{*}{$\begin{array}{l}\text { Follthlatm Marker } \\
\text { Locus: (D5 5474) }\end{array}$} & $\begin{array}{l}\text { Chromosome } \\
5: 53,400,409- \\
53,487,134\end{array}$ & Homo & PI9883 & PI9883 & \\
\hline & & Sapiens & & & \\
\hline Insulin receptor & $\begin{array}{l}\text { Chromosome 19: } \\
7,1 \text { I2,255-7,294,034 }\end{array}$ & Homo & P062I3 & AAA59452 & IGAG \\
\hline (Marker Locus: MISR) & & Sccoiens & & & \\
\hline Insulin receptor & $\begin{array}{l}\text { Chromosome } \\
\text { 19:4,903,0804,962,154 }\end{array}$ & Homo & 096T88 & 96188 & 2.FAZ \\
\hline $\begin{array}{l}\text { (Marker Locus: } \\
\text { DI92216) }\end{array}$ & & \&piens & & & \\
\hline Insulin receptor & $\begin{array}{l}\text { Chromosome 19: } \\
7,595,902-7,618: 304\end{array}$ & Homo & 09PIY5.2 & 09PI Y5 & - \\
\hline $\begin{array}{l}\text { (Marker Locus: } \\
\text { DI92905) }\end{array}$ & & \&pions & & & \\
\hline Insulin receptor & $\begin{array}{l}\text { Chromosome 19: } \\
8,065,402-8,149: 846\end{array}$ & Homo & Q75N90 & Q75N90 & - \\
\hline $\begin{array}{l}\text { (7arker Locus: } \\
\text { DI92884) }\end{array}$ & & Scpions & & & \\
\hline Insulin receptor & $\begin{array}{l}\text { Chromosome } \\
\text { 19:8,520,790-8,577,577 }\end{array}$ & Homo & 160 & 160 & - \\
\hline $\begin{array}{l}\text { (larker Locus: } \\
\text { DI9239I) }\end{array}$ & & Sapiens & & & \\
\hline Insulin receptor & $\begin{array}{l}\text { Chromosome 19: } \\
8,580,242-8,610: 735\end{array}$ & Homo & Q9H324.2 & Q9H324 & - \\
\hline $\begin{array}{l}\text { (Marker Locus: } \\
\text { DI92906) }\end{array}$ & & Sapiens & & & \\
\hline Insulin receptor & $\begin{array}{l}\text { Chromosome 19: } \\
\text { 13,906:201-13,930,879 }\end{array}$ & Homo & Q6PINO & Q6PINO & \\
\hline $\begin{array}{l}\text { (Marker Locus: } \\
\text { DI } 92840 \text { ) }\end{array}$ & & Sapiens & & & \\
\hline Insulin receptor & $\begin{array}{l}\text { Chromosome 19: } \\
\text { |8,207:961-|8,255,419 }\end{array}$ & Homo & Q08493 & Q08493 & ILXIT \\
\hline $\begin{array}{l}\text { (Marker Locus: } \\
\text { DI922I2) }\end{array}$ & & Sapiens & & & \\
\hline $\begin{array}{l}\text { Insulin receptor } \\
\text { (Marker locus: } \\
\text { DI954I0) }\end{array}$ & $\begin{array}{l}\text { Chromosome 19: } \\
\text { I7,281:645-17,287,646 }\end{array}$ & $\begin{array}{l}\text { Homo } \\
\text { sapiens }\end{array}$ & Q8N.A.G6 & 08NAG6 & \\
\hline \multirow[t]{2}{*}{$\begin{array}{l}\text { Insulin receptor } \\
\text { substrate I }\end{array}$} & $\begin{array}{l}\text { Chromosome } \\
2: 226,731317- \\
226,799,759\end{array}$ & Homo & P35568 & NP 5535 & I IRS \\
\hline & & Sapiens & & & \\
\hline \multirow[t]{2}{*}{$\begin{array}{l}\text { Peroxisome } \\
\text { proliferator-activated } \\
\text { receptor-comma }\end{array}$} & $\begin{array}{l}\text { Chromosome } \\
3: 12,287,368- \\
12.434,356\end{array}$ & Homo & P3723I & P3723I & IFM6 \\
\hline & & Sapiens & & & \\
\hline $\begin{array}{l}\text { Mothers against } \\
\text { clecapentaplegic } \\
\text { homolog 4(gene } \\
\text { symbol: MADH4) }\end{array}$ & $\begin{array}{l}\text { Chromosome 18: } \\
\text { 5I,028394-5I,085,045 }\end{array}$ & $\begin{array}{l}\text { Homo } \\
\text { sapiens }\end{array}$ & Q13485 & Q13485.I & $|1:| 13 \mid$ \\
\hline Androgen receptor & $\begin{array}{l}\text { Chromosome X: } \\
67,544,032-67: 730,619\end{array}$ & Homo & PI0275 & PI0275 & IE3G \\
\hline
\end{tabular}


Table Continued

List of 43 Genes involved in PCOS with UNIPROT-ID. PDB-ID and chromosomal locations

\begin{tabular}{|c|c|c|c|c|c|}
\hline (Marker Locus:_AR) & & Sapiens & & & \\
\hline $\begin{array}{l}\text { CYPI IA-cytochrome } \\
\text { P450 side-chain } \\
\text { cleavage enzyme }\end{array}$ & $\begin{array}{l}\text { Chromosome I5: } \\
\text { 74,337:759-74,367,740 }\end{array}$ & Homo & P05 I08 & P05 I08 & $3 N 9 Y$ \\
\hline $\begin{array}{l}\text { Marker Locus: } \\
\text { (DI55519) }\end{array}$ & & Sccoiens & & & \\
\hline $\begin{array}{l}\text { CYPI7- } \\
\text { Oochrome P450 } \\
\text { I7a-hydroxylasey I7,20- } \\
\text { desmolase }\end{array}$ & $\begin{array}{l}\text { Chromosome } \\
\text { I0: I02,830,53I- } \\
102,837: 533\end{array}$ & Homo & P05093 & P05093 & $2 \mathrm{Cl}$ \\
\hline $\begin{array}{l}\text { Marker Locus: } \\
\text { (DI05I92) }\end{array}$ & & Sccoiens & & & \\
\hline $\begin{array}{l}\text { CY? } 19-0 \text {-chrome } \\
\text { P450 aromatase }\end{array}$ & $\begin{array}{l}\text { Chromosome I5: } \\
\text { 5I,208:057-5I,338,610 }\end{array}$ & Homo & PII5II & PII5II & ITQA \\
\hline Marker Locus: (CYPI9) & & Sccoiens & & & \\
\hline $\begin{array}{l}\text { I7 b-Irydmxysteroid } \\
\text { dehydrogenase, type I }\end{array}$ & $\begin{array}{l}\text { Chromosome 17: } \\
42,549: 2 \text { I4-42,555,2 I3 }\end{array}$ & Homo & PI406I & PI406I & $\mathrm{IA} 27$ \\
\hline $\begin{array}{l}\text { Marker Locus: } \\
\text { (DI75934) }\end{array}$ & & Sccoiens & & & \\
\hline $\begin{array}{l}\text { I7 b-hydroxysteroid } \\
\text { dehydrogenase, type } \\
\text { LE Marker Locus: } \\
\text { (HSDI7B2) }\end{array}$ & $\begin{array}{l}\text { Chromosome 16: } \\
82,035: 004-82,098,534\end{array}$ & Homo & P37059 & P37059 & - \\
\hline & & \&piens & & & \\
\hline $\begin{array}{l}\text { I7 b-hydmxvsteroid } \\
\text { dehydrogenase, type } \\
\text { DI Marker Locus: } \\
\text { (99SI809) }\end{array}$ & $\begin{array}{l}\text { Chromosome 9: } \\
96,235,306-96: 302,152\end{array}$ & Homo & P37058 & P37058 & - \\
\hline
\end{tabular}

Historical concepts about the correlation between PCOS and some genes like follistatin and lamin failed to act as a definitive diagnostic tool for the disease. None of these genes are conclusive for PCOS diagnosis, even though most of them were found in high prevalence among PCOS patients. Some of them have a known role in the disease while other's role still ambiguous. The non-specificity and the overlapping symptoms between PCOS and other endocrinal abnormalities form a huge obstacle which the investigator must overcome to find a valuable genetic marker for PCOS.

Alteration in enzymes function either by inhibition or lack of function can affect the normal function of the body. Therefore, enzyme alteration in ovaries can lead to many symptoms; one of which is polycystic ovarian syndrome (PCOS). As discussed earlier, theca cells from polycystic ovaries show a significant increase in both androstenedione and progesterone production in vitro when compared to normal theca. ${ }^{18}$ This suggests that the putative defect in steroidogenesis occurs at or above the level of progesterone biosynthesis. The cholesterol side-chain cleavag enzyme, cytochrome P450scc, catalyses the conversion of cholesterol to pregnenolone, the first rate-limiting step at the start of the steroid hormone biosynthesis pathway. ${ }^{19,20}$ It is hypothesised that upregulation of this enzyme could lead to an increase in androgen production.
A second steroidogenic enzyme, aromatase (cytochrome P450arom), catalyses the conversion of the $\mathrm{C}_{19}$ steroids (androgens) to the $\mathrm{C}_{18}$ estrogens. Evidence for altered regulation of this enzyme in PCOS comes from the observation that granulosa cells from anovulatory polycystic ovaries are hyper-responsive to FSH in vitro, displaying significantly greater estradiol production compared to granulosa cells from normal ovaries. ${ }^{21}$ Conversely, two other studies have demonstrated cases in which aromatase deficiency was associated with hyperandrogenemia. ${ }^{22,23}$ These studies also suggest that an altered regulation of this enzyme may be involved in PCOS.

A study was conducted to investigate P450scc and P450arom enzymes as potential candidates for involvement in PCOS/MPB. The genes encoding P450scc (CYP11a) and P450arom (CYP19) are both located on the long arm of chromosome 15 at positions $15 \mathrm{q} 24$ and 15 q21.1 respectively. Given uncertainties regarding the segregation model for PCOS, both parametric and non-parametric linkage analyses were conducted in 20 PCOS/MPB pedigrees. Mutation screening of the promoter region of CYP11a was also carried out. Finally, using a CYP11a intragenic marker, an association study in a case-control data set of consecutively identified Europid women with PCOS and matched controls was carried out. Both parametric and non-parametric linkage analyses were carried out for each of the candidate loci using 
the GENEHUNTER program. ${ }^{24}$ The position and chromosomal order of the microsatellite markers was used in the analyses. Clear exclusion of the CYP19 locus was obtained as there was no evidence for excess allele sharing across the whole region.

The non-parametric results for the CYP11 a locus provided evidence for excess allele sharing. The results from these data suggested that $\sim 60 \%$ of the pedigrees may be linked to the CYP11a (ac) $)_{\mathrm{n}} / \mathrm{D} 15 \mathrm{~S} 169$ locus.

Since any pathogenic mutations in CYP11 $a$ are expected to lead to upregulation, mutation screening of the promoter region of CYP11a was carried out. This region is believed to contain multiple cAMPregulated elements, responsible for increasing basal transcriptional activity, and elements involved in repression of expression. ${ }^{25}$ PCR amplification, followed by direct sequencing analysis, was used to screen $1.85 \mathrm{~kb}$ of sequence in ten affected and eight unaffected members of the pedigrees. No alterations to the published sequence were found in any individual apart from variation in the number of repeat units of the two microsatellite polymorphisms present in this region [a dinucleotide (ac) repeat, and a pentanucleotide (ttta) repeat, at positions -1376 and -528 respectively from the ATG start of translation site]. Individuals in the case-control study were typed for the CYP11a (ttta) marker. Four alleles were observed in our data set, alleles 216, 226, 236 and 241 with observed frequencies of 0.59 , $0.28,0.04$ and 0.09 respectively. In order to reduce multiple allele comparisons for infrequent alleles, individuals were allocated to two groups according to their genotype, those having at least one copy of the common, four-repeat unit alleles (designated 216+) and those with no 216 allele (designated 216-). An association between this polymorphism and serum testosterone levels was found in the total data set. The genotype distributions were compared of each quartile of serum testosterone level within the total data set, and the proportion of individuals with 216- genotypes in each quartile were (from highest to lowest) $0.44,0.23,0.18$ and 0.13 .

On subgroup analysis, the association between CYP11a genotype and testosterone was only evident in the women with PCOS. There was no significant difference between the mean testosterone levels of 216- and 216+ individuals for either the normal controls or the a PCO groups. However, the numbers in these groups were small, raising the possibility of error.

Next, the CYP11 a genotype distributions were compared between subject groups. Since the association between serum testosterone levels and CYP11a genotype was only seen in the PCOS group, taking the entire data-set (including those without testosterone levels available), compared PCOS women with a combined normal control/a PCO group, and a significant difference in the distribution of genotypes between these groups was observed.

The data showed that the gene coding for aromatase (CYP19) can be excluded as a major determinant of risk for PCOS/MPB. In contrast, the linkage results for CYP11 a (encoding side chain cleavage) provide support for the involvement of this gene in the etiology of PCOS. The association data demonstrated that allelic variants of CYP 11 a mediate the development of hyperandrogenemia, which is in turn associated with PCOS and hirsutism. The association of this gene with hirsutism and the lack of association with ovulatory status indicates that CYP11 a predominantly has a role in the development of hirsutism in PCOS.

A significant association had been shown between alleles of the CYP11a (tttta) ${ }_{n}$ marker and serum testosterone levels. It is feasible that allelic variants of this rate-limiting enzyme could influence the level of androgen production. This association however, can only be demonstrated in the PCOS group. The failure to demonstrate association in the aPCO (women with polycystic ovaries without any symptoms; silent) and normal groups may be due to small sample sizes. Alternatively, it may be that this locus has a modifying effect, interacting with other genetic and/or environmental factors controlling the development of the ovarian changes and thereby influencing the clinical presentation of PCOS.

CYP11a (tttta) is present in the promoter region of CYP11a and sequencing analysis of the surrounding regions does not reveal any other mutations likely to have direct pathogenic effects. However, since only $1.85 \mathrm{~kb}$ of sequence was screened, the putative mutation could be located outside this region. Recently many gene-associated VNTRs (variable number tandem repeats) have been implicated in the regulation of the genes, such as the insulin gene VNTR (30). Also, direct association between alleles of a (ttta) $)_{n}$ repeat polymorphism, present in the $5^{\prime}$ flanking region of the apolipoprotein(a) gene, and plasma levels of $\mathrm{Lp}(\mathrm{a})$ has been demonstrated. ${ }^{26}$ Expression studies have shown a 5-fold higher transcriptional activity in constructs containing eight copies of the apolipoprotein(a) (tttta) repeat compared to those with nine copies. ${ }^{26}$

However, no regulatory function has been assigned to the CYP11a (tttta) polymorphism, and further investigation of the promoter region of CYP11a is required to determine the relationship between the (tttta) repeat polymorphism and the regulation of this gene.

Allelic variants of CYP11a have a role in the etiology of hyperandrogenemia. This in turn may be sufficient to cause the altered ovarian morphology or may act in conjunction with other genetic factors to modify the phenotypic expression of PCOS.

It is not enough to use genetic markers or enzyme for definitive diagnosis of PCOS, as is evidenced by the fact that gene expression varies from individual to individual, and that the genes may manifest differently in aPCO individuals and PCOS patients. Moreover, different factors such as environment also control the phenotype.

Many different protein markers are currently assessed in PCOS individuals, although most of them are used for prognosis, monitoring and to prevent further complications, such as cardiovascular disease.

The pathogenesis of PCOS can be associated with elevated gut permeability. Obesity, irritable bowel syndrome and chronic fatigue syndrome are linked to an increase in gut permeability and contribute to the development of the disease. Regulation of gut permeability is reversibly regulated by Zonulin, a physiological mediator found in the counterpart of vibrio cholera zonula. This non-invasive biomarker is used to indicate gut permeability as it is increased in the small intestine and related to intestinal immunity. Its role in the indication of metabolic diseases has been recently recognized. ${ }^{27}$ To confirm the correlation between high levels of serum zonulin and the development of PCOS, anthropometric data were collected from the control and the experimental group, and the concentration of serum zonulin was measured by ELISA (sensitivity $<0.01 \mathrm{ng} / \mathrm{ml}$ ). It has been confirmed that high levels of serum zonulin correspond to PCOS, and a strong relationship has been demonstrated between increased levels of serum zonulin and insulin resistance, dyslipidemia, and the severity of menstrual disorders. This study correlated the significance of gut permeability and PCOS, and reported that zonulin is elevated in PCOS group compared to non-PCOS participants; as speculated, gut permeability alterations result in compromising the intestinal barrier and facilitating the access of infectious agents leading to 
overproduction of gamma interferon and interleukin-6, which in turn control the production of zonulin. Although several studies have been conducted to demonstrate the role of serum zonulin as a biomarker in the diagnosis of PCOS, the mechanistic link between the development of PCOS and zonulin remain unclear. This imitation can be improved by the measurement of cytokines such as b- 1 interleukin, interleukin-6, and tumor necrosis factor. ${ }^{28}$

The visceral adipose tissue is one of the sites responsible for the secretion of adiponectin, an adipocytokine produced by mature adipocytes. It is known that the levels of serum adiponectin go down in obese individuals which leads to an increase in their insulin resistance, thus, developing polycystic ovary syndrome. ${ }^{29}$ The marker adiponectin and insulin resistance have been correlated to be associated with type 2 diabetes..$^{18}$ A cross sectional observational research has been conducted to determine the degree of insulin resistance in patients with PCOS by measuring the level of serum adiponectin in urine samples via ELISA. ${ }^{30}$ It has been confirmed that there is relation between the concentration of serum adiponectin detected and the degree of insulin resistance in PCOS patients; decreased adiponectin levels reversely correlate with an increased degree of insulin resistance and BMI in PCOS patients. As discussed earlier, multiple metabolic diseases are hidden under polycystic ovary syndrome, the most significant ones being hyperinsulinemia and insulin resistance. In the above-mentioned study, it has also been demonstrated that type 2 diabetes mellitus in patients with PCOS is mainly due to decreased levels of serum adiponectin, as it increases their insulin sensitivity and obstructs the function of beta cells. Further, it confirmed that obese PCOS patients have lower levels of serum adiponectin compared to the group who exhibit normal weight. Despite the pathogenesis of PCOS still being debatable and controversial, it has been observed that low levels of adiponectin, hyperinsulinemia and hyperandrogenism contribute to the development of the disease.

Serum adiponectin level is considered an adequate biomarker in the diagnosis of PCOS and the degree of insulin resistance. It also aids doctors in prognosis and prediction of further complications, such as systemic morbidity of PCOS..$^{20}$

Leptin, tumor necrosis factor, alpha-interferon, ghrelin, retinol bindingprotein-4, resisten, visfatin and apelin are other molecules produced by the adipose tissue. These act as mediators involved in lipid metabolism atherosclerosis, immunity and inflammation, insulin sensitivity and many physiological processes. ${ }^{31}$

Apelin, an adipokine secreted by the adipose tissue as a biologically active peptide, has been identified from the extracts of bovine stomach in both human and mice. It has been considered a marker for the diagnosis of PCOS since recent times, as it is involved in the pathogenesis of cardiovascular diseases and dyslipidemia. It is connected to insulin resistance with other markers: tumor necrosis factor, interleukin-6, C-reactive protein, Insulin-like growth factor and sex steroids. Apelin can be found in multiple organs including heart, brain, kidneys and lungs. It functions as an endogenous receptor ligand. Different levels and concentrations of serum apelin are associated with different stages of ovarian follicles. This biomarker affects water intake and hypothalamo-hypophyseal axis and the cardiovascular system. ${ }^{32}$ The conducted enzyme immune assay method was used to determine the level of plasma apelin, and it has been found that it is higher in PCOS patients compared to the control group: the increased concentrations of plasma apelin can be associated with androgenic obesity, hypothalamo-hypophyseal axis, polycystic ovaries, increased hip to waist ratio and in the case of impairment between LH/FSH interaction. ${ }^{33}$ However, due to the lack of sufficient data and studies regarding this adipokine, it cannot be used as a definitive diagnostic marker and other adipokines with similar properties are used instead, like visfatin and alpha tumor necrosis factor. ${ }^{18}$

Although the pathogenesis of PCOS is somehow unclear, it is well known that it is caused by impaired folliculogenesis leading to oligo-ovulatory cycles, due to which high levels of Anti-Mullerian Hormone (AMH) are produced. AMH is secreted by granulosa cells found in the antral follicles, as part of the normal body physiological process, AMH should be decreased following the development of follicles. However, the opposite can happen due to pre-antral follicle proliferation and high production of follicular secretions which leads to PCOS. The discovery of AMH has avoided the need for invasive follicular phase ultrasound examination, because the detection of high levels of this biological marker is adequate in assessing an accurate diagnosis. It is coupled with other clinical features such as, mean ovarian volume, the duration of menstrual cycle and levels of serum aldosterone and testosterone. ${ }^{34}$

To rule out the significant role of AMH as a biological maker in diagnosing PCOS, an experiment was conducted to evaluate the levels of AMH in both PCOS patients and healthy individuals, where the AMH was measured by an enzymatically amplified two-sided immunoassay with a sensitivity of $0.006 \mathrm{ng} / \mathrm{ml} .{ }^{25}$ It was found that women with PCOS have triple the amount of serum AMH compared to healthy women, and these results are compatible with the results of several studies conducted previously that reported higher levels of $\mathrm{AMH}$ in PCOS women.

It can, therefore be concluded that in cases of polycystic ovary syndrome, the levels of AMH, in addition to LH/FSH ratio increase. However, LH/FSH ratio can be within the normal range in approximately $80 \%$ of patients presented with PCOS. Thus, AMH is considered the strongest marker for diagnosis and may challenge the need for other invasive and time-consuming examinations, such as ultrasound. This marker might especially help in cases of adolescents and women in the reproductive age, as ultrasound examination is not possible due to their virginal status. Moreover, physicians will get a very poor quality of the images produced by ultrasonography in obese PCOS patients. Other uses of AMH include the prediction of body response to treatment and prescribing the appropriate dose of gonadotropin.

Testosterone and androsterenedione are produced by the ovaries and the adrenal glands, and the enzyme 5a-reductase converts them to dihydrotestosterone and tetrahydrocortisol. Since metabolites and byproducts of this steroid hormone have negative consequences and result in other metabolic diseases such as liver disease and insulin resistance, multiple steroid hormones should be quantified, and their relation to each other should be studied. To detect female hyperandrogenism, testosterone is measured in routine clinical practice. However, if testosterone is the sole clinical investigator, the detection of excess androgen can be missed. ${ }^{35}$ Moreover, in some PCOS patients, the level of testosterone can be normal, but androstenedione is relatively high. It was investigated that the ratio of testosterone to dihydrotestosterone is an adequate co-measurement that helps in the diagnosis of PCOS. Mass spectrometry and immunoassays were used to measure the level of testosterone to dihydrotestosterone.

J. Munzker and colleagues conducted one of the most sophisticated studies, in which they measured the ratio of testosterone to dihydrotestosterone on well characterized and standardized PCOS patients, and carried out a detailed assessment. They used liquid 
chromatography/mass spectrometry for a comprehensive measurement of steroid hormones coupled with anthropometric parameters, as these methods showed high sensitivity and specificity, and helped in the quantification and evaluation of hormones. ${ }^{26}$ Hyperandrogenism is a key factor that helps in understanding of PCOS, and one way to evaluate it is through the measurement of multiple steroid hormones and their relation to each other. So, the ratio of testosterone to dihydrotestosterone is one of the novel biomarkers in the diagnosis of PCOS.

Copeptin is another novel biomarker used in the diagnosis of PCOS. It resembles arginine vasopressin hormone found in human and mice. It is known to stabilize vascular tone and fluid balance. Due to the difficulty in measuring arginine vasopressin, copeptin is used as it is more stable and sensitive. It has the same C-terminal part as arginine vasopressin, composed of a glycopeptide of 39 amino acids. Enzyme immunoassay is used to measure the levels of copeptin via semi-quantitative method, with a sensitivity of $0.09 \mathrm{ng} / \mathrm{mL}$. The conducted experiment showed a significant difference in copeptin levels in PCOS patients compared to healthy individuals, suggesting that it can be used a new non-invasive diagnostic tool for PCOS. This biomarker has a positive association with insulin resistance and cardiometabolic complications as well. Different concentrations of copeptin are correlated with the different stages of polycystic ovary syndrome, and can be used to predict the consequences of the disease. $^{18}$

Irisin is a biomarker that was discovered to be responsible for the development of white fat and glucose tolerance recently. It is a myokine found circulating in serum and plasma of PCOS patients. However, sufficient research done regarding this biomarker has not been carried out, as it is unstable and easily degraded. So, it cannot be considered as a definitive marker in diagnosis of PCOS yet; more characterized and well-designed experiments have yet to be demonstrated. However, according to the conducted meta-analysis, there is a relation between circulating irisin and PCOS; it has been found to be higher in the experimental group compared to the control group, and it is interconnected with insulin resistance. ${ }^{18}$ It has also been suggested that the increase in irisin level might be due to other factors, such as metabolic stress. Irisin is also affected by drugs (metformin), obesity and high free androgen index (FAI). It has been noted that elevated levels of circulating irisin is associated with insulin resistance, hyperandrogenemia and metabolic syndrome.

Ghrelin is a hormone that influences pituitary gland function by secreting hormones. It is also associated with energy homeostasis, cell proliferation and replication. It is a glycopeptide that possesses multifunctions, and is produced by the gastric. There is another hormone coded by the same gene as ghrelin, which is obestatin. ${ }^{36}$ The levels of these two hormones are known to be changed in cases of PCOS, due to disorders in pituitary hormone secretions. For PCOS to be diagnosed based on these two markers, a specific ratio and heir relation to each other should be defined. Radioimmunoassay is the method of choice in measuring the concentration of ghrelin and obestatin in the serum of both experimental group (patients with PCOS) and a control group. A specific chemiluminescence assay with high specificity is used to measure LH/FSH. It was elucidated that in cases of PCOS, the level of ghrelin increases, in comparison to obestatin, which decreases. The incompatibility of these two markers is negatively related to LH/FSH. It has also been concluded that obestatin and ghrelin contribute to the pathogenesis of PCOS. ${ }^{26}$

Metastin is a hormone that is involved in multi-metabolic diseases including polycystic ovary syndrome. It is also called Kisspeptin. KISS-1 is a gene that codes for this hormone at the time of puberty. ${ }^{18} \mathrm{It}$ induces the production of gonadotrophin releasing hormone (GnRH), which in turn plays a role in ovulation. It is found in males (in testes), and in placenta, small intestine and pancreas. It is illustrated that metastin hormone tends to be dimorphic in females, but not in males. Increased levels of metastin affect the level of lutenizing hormone (LH) by increasing it. It is elucidated that the levels of metastin hormone rises in non-obese PCOS patients, which can be used as a diagnostic marker. ${ }^{37}$

It has also been observed recently that there is a correlation between haptoglobin and PCOS by proteomic studies. ${ }^{38} \mathrm{Hp}$ is a positive marker of inflammation that prevents oxidative damage by binding to any free hemoglobin. Since oxidative stress and inflammation are involved in the development of PCOS, it only makes sense that haptoglobin levels are increased in PCOS patients.

Although there are many proteins which are potential biomarkers for PCOS, there has been insufficient research done upon them due to their instability and easily degradable nature. Moreover, most of them provide useful information for prognosis and disease monitoring rather diagnosis. It would, therefore, be most useful to combine various markers, and assess them together for a definitive diagnosis.

\section{Conclusion}

PCOS is the most prevalent, complex endocrine disorder of the female reproductive system that is influenced by biochemical, genetic and environmental factors, and can even lead to long term morbidities, such as diabetes and cardiovascular disease. PCOS patients often present with hirsutism, sudden weight gain, hormone imbalances, oligo/anovulation, polycystic ovaries, hyperinsulinemia, hyperandrogenemia, and higher levels of testosterone. Although there is no definitive pathophysiology for the disorder, the primary defects are attributed to insulin resistance, and luteinizing hormone and follicle-stimulating hormone levels, which leads to excess androgen and testosterone production; higher levels of LH than FSH also leads to anovulation or oligo-ovulation and problems with conception.

PCOS is diagnosed in patients by excluding all other possible endocrinopathies, following the presence of androgenemia, androgenism and an/oligoovulation. Although some places rely on the appearance of ovaries for diagnosis, various gene, and protein and enzyme markers can also be used. The genetic markers investigated are the ones associated with regulating insulin function and androgen synthesis, and 43 genes isolated have been identified to be related to PCOS. However, these genetic markers are not useful for early diagnosis, and can only be used to confirm PCOS in patients already exhibiting the definitive symptoms. Protein and enzyme markers are commonly used for prognosis and patient monitoring to prevent the development of the complications of PCOS, such as CVD. Proteins of the adipose tissue have been found to be greatly related to insulin resistance and the development of PCOS. However, the instability and easily degradable nature of enzymes and proteins have prevented adequate research from being carried out on them. The diagnosis of PCOS, therefore, relies on the analysis of multiple factors.

Currently, metformin is prescribed to PCOS patients as it has been observed to regulate menstruation and increase the chances of conception in both androgenic and non-androgenic forms of the syndrome. ${ }^{39}$ A recent approach suggests that the administration of myo-inositol, gymnemic acid and L-methylfolate shows a significant decrease in testosterone levels, especially in obese/overweight 
patients. ${ }^{40}$ Above all, as there is a decreased metabolism in PCOS patients, they are advised to maintain an active lifestyle to prevent the development of obesity, diabetes, CVD and other morbidities.

\section{Acknowledgments}

None.

\section{Conflicts of interest}

Authors disclose no conflict of interests in publication of this study.

\section{References}

1. Azziz R, Woods KS, Reyna R, et al. The prevalence and features of the polycytic ovary syndrome in an unselected population. J Clin Endocrinol Metab. 2014;89(6):2745-2749.

2. Broekmans FJ, Knauff EA, Valkenburg O, et al. PCOS according to the Rotterdam consensus criteria: change in prevalence among WHO-II anovulation and association with metabolic factors. BJOG. 2016;113(10):1210-1217.

3. Goodarzi MO, Dumesic DA, Chazenbalk G, et al. Polycystic ovary syndrome: etiology, pathogenesis and diagnosis. Nat Rev Endocrinol. 2012;7(4):219-231.

4. Stepto Nigel K, Cassar S, Joham AE, et al. Women with polycystic ovary syndrome have intrinsic insulin resistance on euglycaemichyperinsulaemic clamp. Hum Reprod. 2013;28(3):777-784.

5. Azziz Ricardo, Enrico Carmina, ZiJiang Chen, et al. Polycystic ovary syndrome. Nature Reviews Disease Primers. 2016.

6. Jayasena CN, Franks S. The management of patients with polycystic ovary syndrome. Nat Rev Endocrinol. 2014;10(10):624-636.

7. Rajkhowa M, Brett S, Cuthbertson DJ, et al. Insulin resistance in polycystic ovary syndrome is associated with defective regulation of ERK $1 / 2$ by insulin in skeletal muscle in vivo. Biochem J. 2009;418(3):665-671.

8. Goodarzi MO, Dumesic DA, Chazenbalk G, et al. Polycystic ovary syndrome: etiology, pathogenesis and diagnosis. Nat Rev Endocrinol. 2011;7(4):219-231.

9. Merkin SS, Phy JL, Sites CK, et al. Environmental determinants of polycystic ovary syndrome. Fertil Steril. 2016;106(1):16-24.

10. Diamanti-Kandarakis E, Bourguignon JP, Giudice LC, et al. Endocrinedisrupting chemicals: an endocrine society scientific statement. Endocr Rev. 2009;30(4):293-342.

11. Panda PK, Rane R, Ravichandran R, et al. Genetics of PCOS: A systematic bioinformatics approach to unveil the proteins responsible for PCOS Genom Data. 2016;8:52-60.

12. Nuzhat Shaikh, Roshan Dadachanji, Srabani Mukherjee. Genetic Markers of Polycystic Ovary Syndrome: Emphasis on Insulin Resistance. International Journal of Medical Genetics. 2014.

13. Urbanek M, Legro RS, Driscoll DA, et al. Thirty-seven candidate genes for polycystic ovary syndrome: Strongest evidence for linkage is with follistatin. Proc Natl Acad Sci U S A. 1999;96(15):8573-8578.

14. Dunaif A, Wu X, Lee A, et al. Defects in insulin receptor signaling in vivo in the polycystic ovary syndrome (PCOS). Am J Physiol Endocrinol Metab. 2001;281(2):E392-E399.

15. Gharani N, Waterworth DM, Batty S, et al. Association of the steroid synthesis gene CYP11a with polycystic ovary syndrome and hyperandrogenism. Hum Mol Genet. 1997;6(3):397-402.
16. Eisenberg I, Nahmias N, Novoselsky Persky M, et al. Elevated circulating micro-ribonucleic acid (miRNA)-200b and miRNA-429 levels in anovulatory women. Fertil Steril. 2017;107(1):269-275.

17. Sørensen AE, Wissing ML, Salö S, et al. MicroRNAs Related to Polycystic Ovary Syndrome (PCOS). Genes. 2014;5(3):684-708.

18. Gilling-Smith C, Willis DS, Beard RW, et al. Hypersecretion of androstenedione by isolated thecal cells from polycystic ovaries. $J$ Clin Endocrinol Metab. 1994;79(4):1158-1165.

19. Stone D, Hechter O. Studies on ACTH action in perfused bovine adrenals: aspects of progesterone as an intermediary in corticosteroidogene-sis. Arch Biochem Biophys. 1955;54(1):121-128.

20. Halkerston ID, Eichhorn J, Hechter O. A requirement for reduced triphosphopyridine nucleotide for cholesterol side-chain cleavage by mitochondrial fractions of bovine adrenal cortex. J Biol Chem. 1961;236:374-380.

21. Mason HD, Willis DS, Beard RW, et al. Estradiol production by granulosa cells of normal and polycystic ovaries: relationship to menstrual cycle history and concentrations of gonadotropins and sex steroids in follicular fluid. J Clin Endocrinol Metab. 1994;79(5):1355-1360.

22. Harada N, Ogawa H, Shozu M, et al. Genetic studies to characterize the origin of the mutation in placental aromatase deficiency. Am J Hum Genet. 1992;51(3):666-672.

23. Ito $\mathrm{Y}$, Fisher $\mathrm{CR}$, Conte FA, et al. Molecular basis of aromatase deficiency in an adult female with sexual infantilism and polycystic ovaries. Proc Natl Acad Sci U S A. 1993;90(24):11673-11677.

24. Kruglyak L, Daly MJ, Reeve-Daly MP, et al. Parametric and nonparametric linkage analysis: a unified multipoint approach. Am J Hum Genet. 1996;58(6):1347-1363.

25. Moore CC, Brentano ST, Miller WL. Human P450scc gene transcription is induced by cyclic AMP and repressed by 12-O-tetradecanoylphorbol13-acetate and A23187 through Independent cis elements. Mol Cell Biol. 1990;10(11):6013-6023.

26. Mooser V, Mancini FP, Bopp S, et al. Sequence polymorphisms in the $\mathrm{Apo}(\mathrm{a})$ gene associated with specific levels of $\mathrm{Lp}(\mathrm{a})$ in plasma. Hum Mol Genet. 1995;4(2):173-181.

27. Zhang D, Zhang L, Yue F, et al. Serum zonulin is elevated in women with polycystic ovary syndrome and correlates with insulin resistance and severity of anovulation. Eur J Endocrinol. 2015;172(1):29-36.

28. Polak K, Czyzyk A, Simoncini T, et al. New markers of insulin resistance in polycystic ovary syndrome. J Endocrinol Invest. 2017;40(1):1-8.

29. Lau C-H, Muniandy S. Novel adiponectin-resistin (AR) and insulin resistance (IRAR) indexes are useful integrated diagnostic biomarkers for insulin resistance, type 2 diabetes and metabolic syndrome: a case control study. Cardiovasc Diabetol. 2011;10:8.

30. Artimani T, Saidijam M, Aflatoonian R, et al. Downregulation of adiponectin system in granulosa cells and low levels of HMW adiponectin in PCOS. J Assist Reprod Genet. 2016;33(1):101-110.

31. Energin H, Kaba M, Ugur M. Serum adiponectin level and clinical, metabolic, and hormonal markers in patients with polycystic ovary syndrome. Int J Fertil Steril. 2014;7(4):331-336.

32. Sun X, Wu X, Zhou Y, et al. Evaluation of apelin and insulin resistance in patients with PCOS and therapeutic effect of drospirenone-ethinylestradiol plus metformin. Med Sci Monit. 2015;21:2547-2552.

33. Gören K, Sağsöz N, Noyan V, et al. Plasma apelin levels in patients with polycystic ovary syndrome. J Turk Ger Gynecol Assoc. 2012;13(1):27-31. 
34. Deveer M, Deveer R, Basaran O, et al. Serum copeptin, pentraxin 3, anti-mullerian hormone levels with echocardiography and Carotid artery intima-media thickness in adolescents with polycystic ovary syndrome. $J$ Clin Med Res. 2015;7(12):989-994.

35. Münzker J, Hofer D, Trummer C, et al. Testosterone to dihydrotestosterone ratio as a new biomarker for an adverse metabolic phenotype in the polycystic ovary syndrome. J Clin Endocrinol Metab. 2015;100(2):653660

36. Cowan E, Burch $\mathrm{KJ}$, Green $\mathrm{BD}$, et al. Obestatin as a key regulator of metabolism and cardiovascular function with emerging therapeutic potential for diabetes. Br J Pharmacol. 2016;173(14):2165-2181.

37. Clarke H, Dhillo WS, Jayasena CN. Comprehensive review on kisspeptin and its role in reproductive disorders. Endocrinol Metab (Seoul). 2015;30(2):124-141.
38. Carvalho LM, Ferreira CN, Oliveira DK, et al. Haptoglobin levels, but not Hp1-Hp2 polymorphism, are associated with polycystic ovary syndrome. $J$ Assist Reprod Genet. 2017;34(12):1691-1698.

39. Naidukova AA, Ananyev EV, Chernukha GE. Effect of metformin on the reproductive function of women with different phenotypes of polycystic ovary syndrome. Obstetrics and Gynecology. 2017.

40. Stracquadanio M, Ciotta L, Palumbo MA. Effects of myo-inositol, gymnemic acid, and L-methylfolate in polycystic ovary syndrome patients. Gynecol Endocrinol. 2018;34(6):495-501. 